\title{
Médiévales
}

Langues, Textes, Histoire

77 | automne 2019

Mathématiques. Savoirs et enseignements (OrientOccident)

\section{John BLAIR, Building Anglo-Saxon England}

Princeton (NJ), Princeton University Press, 2018, xxiv-472 p.

\section{Alban Gautier}

\section{(2) OpenEdition}

\section{Journals}

Édition électronique

URL : https://journals.openedition.org/medievales/10626

DOI : $10.4000 /$ medievales. 10626

ISSN : 1777-5892

Éditeur

Presses universitaires de Vincennes

Édition imprimée

Date de publication : 5 décembre 2019

Pagination : 177-180

ISBN : 978-2-37924-061-4

ISSN : 0751-2708

Référence électronique

Alban Gautier, « John BLAIR, Building Anglo-Saxon England», Médiévales [En ligne], 77 | automne 2019,

mis en ligne le 12 mai 2020, consulté le 22 avril 2022. URL : http://journals.openedition.org/ medievales/10626; DOI : https://doi.org/10.4000/medievales.10626 
moyen de se distinguer en ville et il ne donne pas naissance à un milieu social distinct et homogène. À Lille et à Dijon, il n'y a pas de «trahison des bourgeois » : le service du prince ne se substitue pas à l'exercice du pouvoir municipal qui reste prestigieux. Les liens entre le duc et la ville reposent ainsi sur des individus qui se positionnent en intermédiaires et qui forment un réseau que les villes peuvent activer au besoin pour solliciter l'attention et la bienveillance du prince.

C'est donc une enquête riche et stimulante que mène Cécile Becchia dans cet ouvrage, dont le grand mérite est de révéler avec rigueur les mécanismes subtils qui constituent le lien fort unissant le prince à sa ville. Grâce à une démarche comparatiste fructueuse - agrémentée de regards sur la situation d'autres villes de l'ensemble bourguignon -, l'auteure met à jour les dynamiques qui sous-tendent le milieu complexe des élites dirigeantes, une démarche qui ravira les chercheurs qui s'intéressent de près ou de loin aux sociétés urbaines médiévales.

Adrien CARBonnet (Sorbonne Université/Centre Roland Mounier - UMR 8596)

John BlaIR, Building Anglo-Saxon England, Princeton (NJ), Princeton University Press, 2018, xxiv-472 p.

Le dernier livre que nous offre John Blair, professeur d'histoire et archéologie médiévales à Oxford, est d'abord un très bel objet. Cinq cents pages reliées sur papier glacé, présentées sous une belle jaquette reprenant une scène de chantier tirée du «Psautier Harléien » ( $\mathrm{XI}^{\mathrm{e}}$ siècle), contiennent un texte dense, mais d'une lecture toujours claire et agréable, une bibliographie de près de mille références et un index très complet, avec noms propres et matières. Surtout, le propos est agrémenté de plus de cent cinquante illustrations en noir ou en couleurs (cartes, dont la plupart sont originales, plans de sites et de bâtiments, schémas, pages de manuscrits, photographies) : un soin extrêmement bienvenu a été apporté à l'ensemble de ces images, par exemple en fournissant de nouveaux dessins à la même échelle de tous les plans archéologiques dès lors qu'ils sont présentés en vis-àvis sur une même page. La lecture de ce livre est donc dès l'abord un plaisir esthétique, qui ne fait qu'augmenter au fur et à mesure que le lecteur découvre combien, sous cette apparence agréable, le propos est intelligent, toujours très bien informé, et en fin de compte très original sans cesser d'être équilibré.

Comme le titre l'annonce, l'auteur cherche à comprendre comment l'Angleterre anglo-saxonne (c'est-à-dire l'Angleterre du haut Moyen Âge, entre le VII et le XI ${ }^{\mathrm{e}}$ siècle) a été « construite». Faisant dialoguer les sources écrites (narratives et documentaires), l'iconographie, la toponymie et surtout l'archéologie de l'habitat et du paysage dont les développements ont été considérables au cours des trois dernières décennies, John Blair tisse ensemble plusieurs enquêtes. Il étudie d'abord l'histoire de la naissance des paysages agraires identifiés comme typiquement anglais par l'historiographie, en particulier celle des openfields dont la localisation prend en écharpe le territoire, du Yorkshire oriental au Dorset. Il s'agit aussi d'explorer la question de l'habitat rural et, dans une moindre mesure, urbain, à la fois pour les élites et pour le reste de la population, qu'il s'agisse de la forme des agglomérations (avec la question de l'émergence de l'habitat groupé) ou de celle des bâtiments (du « hall » au « manoir » en passant par de nombreux autres termes dont la définition a été plus ou moins figée par le discours archéologique et historique). Ces deux questions fondamentales sont articulées avec d'autres, qui reviennent tout au long de l'ouvrage : la structuration politique du territoire et l'organisation spatiale des sites bâtis par les pouvoirs laïcs et ecclésiastiques ; les différences régionales au sein du monde anglo-saxon; les emprunts de l'Angleterre aux espaces voisins, et en particulier au monde scandinave, au risque de négliger quelque peu les parallèles gaulois et surtout 
brittoniques. Ayant lu les travaux des géographes, des anthropologues, mais aussi de ses collègues historiens et archéologues européens dans plusieurs langues, l'auteur se plaît en particulier à proposer des comparaisons avec des sociétés agraires plus récentes, mais dont la structuration sociale, les techniques architecturales et le rapport au paysage peuvent, à son avis, fournir un parallèle pertinent : celles de la Finlande et de la Carélie au $\mathrm{XIX}^{\mathrm{e}}$ et au début du $\mathrm{XX}^{\mathrm{e}}$ siècle.

Pour éclairer toutes ces questions, l'auteur avance quelques hypothèses nouvelles, des modèles explicatifs parfois hardis qui ne manqueront pas de susciter le débat : en cela, John Blair reste fidèle à ses premiers travaux sur l'histoire de l'Église anglo-saxonne, qui l'avaient amené à formuler la minster hypothesis, si discutée dans les deux décennies qui ont suivi sa première apparition ${ }^{2}$. On se contentera de mentionner ici deux idées qui occupent une place essentielle dans l'argumentation de ce nouvel opus. La première consiste à repérer dans un grand nombre de sites d'habitat, mais aussi dans plusieurs paysages agraires, fouillés ou ayant fait l'objet de reconnaissances aériennes ou d'études du parcellaire, des régularités et des alignements qui, selon l'auteur, ne peuvent s'expliquer que par l'usage, à plusieurs reprises au cours de l'histoire anglo-saxonne (surtout au VII et au $\mathrm{X}^{\mathrm{e}}$ siècle), de méthodes d'arpentage au groma, héritées de la tradition romaine et probablement importées depuis le continent européen au vII ${ }^{\mathrm{e}}$ siècle. Sur plusieurs plans inclus dans l'ouvrage sont surimprimées des grilles à l'encre bleu pâle qui dessinent un quadrillage visant, sans gêner la lecture, à faire ressortir l'utilisation systématique d'unités de mesure (selon les régions, la « longue perche» de 5,5 m ou la «courte perche » de 4,6 m). Pour l'auteur, l'utilisation de mesures et de techniques romaines reflète la planification de nombreux habitats à l'initiative des pouvoirs laïcs et surtout ecclésiastiques ayant eu accès à une documentation théorique sur l'arpentage, transmise avec la christianisation. L'évaluation de cette hypothèse appartient à des spécialistes plus qualifiés, mais il importe de noter ici que le lecteur non archéologue, s'il repère en effet sur la plupart de ces plans un souci d'aligner et d'ordonner les éléments de l'habitat, ainsi que l'utilisation évidente de certains modules (par exemple, un double carré de « quatre perches » de côté, qui revient assez souvent) dans les fondations de divers bâtiments, reste dubitatif face à l'idée d'une utilisation systématique de ces mesures dans l'organisation des habitats et la délimitation des parcelles. Bien souvent en effet, les lignes noires (qui sont celles que la prospection a permis d'observer sur le terrain, dans les cadastres ou dans la fouille) ne correspondent guère aux lignes bleues (qui sont celles du grid-planning postulé). Dans certains cas donc, l'utilisation d'une grille saute aux yeux ; mais dans d'autres (par exemple, pour ne citer qu'un seul cas, sur le plan du site de Cowage Farm, fig. 35, p. 122), l'interprétation semble quelque peu forcée. Il manque ici une argumentation plus serrée, que l'auteur a amorcée dans un article et qu'il annonce pour un ouvrage qu'il est en train d'écrire en collaboration ${ }^{3}$. Ajoutons que cette

2. On renverra à trois titres qui ont jalonné ce débat : E. CAMBRIDGE et D. Rollason, «The Pastoral Organization of the Anglo-Saxon Church : A Review of the "Minster Hypothesis" », Early Medieval Europe, 4/1 (1995), p. 87-104 ; J. BLAIR, " Ecclesiastical Organization and Pastoral Care in Anglo-Saxon England », Early Medieval Europe, 4/2 (1995), p. 193212 ; J. BLaIR, The Church in Anglo-Saxon Society, Oxford, 2005. Voir aussi notre article : A. GAUTIER, «La chrétienté anglo-saxonne ( $\left(\mathrm{VII}^{\mathrm{e}}-\mathrm{XI}^{\mathrm{e}}\right.$ siècle) : à propos de quelques publications récentes », Médiévales, 56 (2009), p. 151-168.

3. Il convient donc de lire, en parallèle de ce livre : J. BLAIR, « Grid-Planning in AngloSaxon Settlements : the Short Perch and the Four-Perch Module », dans H. Hamerow éd., Anglo-Saxon Studies in Archaeology and History, 18, Oxford, 2013, p. 18-61. On surveillera la parution prochaine de J. Blair, S. RIPPON et C. SMART, Planning the Early Medieval Landscape, Liverpool. 
hypothèse est articulée à une discussion toponymique qui propose de voir derrière un grand nombre de toponymes en -tun la trace d'une forme de planification; il est dommage que l'argumentation ne tienne pas compte de l'existence dans le nord de la Gaule des nombreux cas strictement parallèles que sont les toponymes en -thun du Boulonnais.

L'autre grande hypothèse du livre tient à la lecture régionalisée de l'histoire anglosaxonne que cherche à promouvoir l'auteur. Elle repose sur un double constat. D'une part, la documentation écrite privilégie certaines régions comme le Kent au vII siècle, la Northumbrie (et en particulier sa partie nord-est) au vIII ${ }^{\mathrm{e}}$ siècle, le Wessex enfin (et dans une moindre mesure les régions immédiatement au nord de la vallée de la Tamise, dans le sud et le sud-ouest de la Mercie) à partir de la fin du $\mathrm{IX}^{\mathrm{e}}$ siècle. Cette observation, qui a déjà été faite de nombreuses fois, est ici mise en rapport avec une autre, beaucoup plus originale : si l'on excepte les sites d'élite, la grande majorité des habitats anglo-saxons fouillés ont précisément été découverts au sein de la zone que dessinent en creux ces régions bien documentées à l'écrit. Plus exactement, les traces d'habitats « anglo-saxons classiques ", construits en bois sur trous de poteaux et/ou tranchées de fondation, ont été majoritairement retrouvées dans une vaste eastern zone dont le cœur est le bassin versant de la Wash et qui s'étend vers le sud-est en direction du Suffolk, vers le nord en direction des deux rives de l'estuaire de la Humber, et par la suite (surtout au $\mathrm{X}^{\mathrm{e}}-\mathrm{XI}^{\mathrm{e}}$ siècle) vers le sud-ouest en direction du West Country. En dehors de cette eastern zone, si l'on écarte quelques grands sites élitaires, 1 'habitat rural reste le plus souvent invisible. Ce décalage met en lumière la difficulté qu'il peut y avoir à faire dialoguer sources archéologiques et sources écrites (par exemple les chartes) quand il s'agit de comprendre l'histoire du paysage et de l'habitat. Il incite aussi l'auteur à écrire pour cette eastern zone, qui recouvre selon les périodes entre un tiers et la moitié de l'Angleterre et qui concentre la majorité de ses propres analyses, une histoire singulière, moins marquée par les contacts avec la Gaule et l'Italie, plus tournée vers la Scandinavie malgré une christianisation aussi précoce qu'ailleurs.

Cette concentration du regard de l'historien sur l'est de l'Angleterre dessine une autre manière de raconter l'histoire du pays tout entier. Elle permet avant tout de proposer une périodisation nouvelle, en quatre temps : deux temps d'accélération des transformations, deux autres où le changement se fait plus lent et cède la place à la stabilisation, certes créative, des structures. Les deux moments-clés sont d'une part le vir siècle, temps de la mise en place des pouvoirs royaux, de la christianisation et surtout de la fondation des institutions religieuses, et d'autre part les trois derniers quarts du $x^{e}$ siècle, qui voient émerger une aristocratie rurale, mais aussi s'amorcer le regroupement de l'habitat et se diffuser le modèle urbain esquissé dès le siècle précédent dans la seule eastern zone. Entretemps, une longue période de consolidation est marquée en particulier par la stabilisation et la montée en puissance de pouvoirs royaux de plus en plus à même de structurer le territoire et d'imposer leur autorité : en cela, l'action des rois merciens Offa et Ceolwulf entre 780 et 820 apparaît encore plus déterminante que celle, plus souvent mise en avant, d'Alfred de Wessex à la fin du $\mathrm{IX}^{\mathrm{e}}$ siècle. Enfin, après l'an 1000, des évolutions qui annoncent à maints égards celles du XII ${ }^{\mathrm{e}}$ et du XIII ${ }^{\mathrm{e}}$ siècle et qui amènent à relativiser la rupture de 1066 - sauf pour quelques phénomènes comme l'essor des châteaux et celui des constructions d'églises en style roman dont le caractère impressionnant a pu amener les historiens de l'architecture à survaloriser l'impact normand sur le paysage et sur l'habitat.

On notera pour finir que l'auteur propose, dans des pages passionnantes, plusieurs hypothèses pour rendre compte de l'invisibilité archéologique des bâtiments où ont nécessairement vécu la plupart des habitants de l'Angleterre avant le $\mathrm{x}^{\mathrm{e}}$ siècle - et en particulier presque tous ceux qui vivaient en dehors de l'eastern zone. Les parallèles ethnographiques scandinaves et finnois s'avèrent singulièrement féconds, puisque ces 
sociétés où ont longtemps dominé la polyculture articulée à l'élevage, l'exploitation de la forêt et l'architecture en bois ont effectivement connu des techniques bien documentées au siècle dernier, qui laissent très peu de traces au sol, avec des constructions légères et mobiles, en planches ou en rondins, parfois simplement posées à terre. L'auteur rappelle que des structures éphémères - tentes, cabanes, pavillons, isbas, etc. - ne sont pas nécessairement synonymes d'inconfort et de pauvreté matérielle : la richesse mobilière peut, dans une large mesure, compenser le caractère rudimentaire des constructions. Ainsi, les great hall complexes du $\mathrm{VII}^{\mathrm{e}}$ siècle, comme ceux qui ont été fouillés à Yeavering, à Cowdery's Down ou récemment à Lyminge, ne doivent pas être vus comme des résidences stables destinées à abriter de manière durable un habitat princier, mais comme des structures plus fugaces qui s'inscrivaient pour un temps, et dans un but d'ostentation, au sein de paysages qu'il s'agissait de contrôler. Plus que les résidences elles-mêmes, ce sont donc les terroirs eux-mêmes qui, à l'instar des central places de l'archéologie scandinave, auraient été les « lieux persistants » des sociétés et des pouvoirs anglo-saxons jusqu'au début du $\mathrm{x}^{\mathrm{e}}$ siècle : dominés par une impressionnante structure préhistorique et/ou romaine (route, tumulus, etc.), émaillés des tombes des ancêtres, pourvus parfois d'une église, délimités par des marqueurs spatiaux bien connus de tous, ils accueillaient des structures ostentatoires en bois que l'on pouvait aisément démonter et reconstruire un peu plus loin, mais toujours au sein du même paysage chargé de signification.

Ce n'est qu'à partir des années 920, et même après 950 en dehors de l'eastern zone, qu'une révolution dans le rapport à l'espace aurait stabilisé à la fois l'habitat d'élite (avec l'émergence de résidences aristocratiques fixes et de plus en plus luxueuses, aux intérieurs divisés en pièces différenciées, parfois pourvues d'enclos tendant vers la fortification), l'habitat rural groupé (avec l'encellulement des populations, l'auteur reprenant ici de manière explicite, avec le terme cellularisation, le concept bien connu dans l'historiographie française) et l'habitat urbain (avec l'apparition des premières structures ayant l'aspect de « rues », comme à York ou à Thetford). Cette révolution n'est pourtant pas « seigneuriale », car les groupes aristocratiques, qui d'après l'auteur sont souvent issus des couches supérieures de la paysannerie, n'exercent pas encore sur leurs voisins une domination coutumière, mais plutôt cette forme d'influence informelle que procurent les liens de clientèle et la capacité qu'ils ont de rendre des services aux communautés d'habitants dans lesquelles, malgré la différenciation croissante dont témoigne leur habitat, ils sont encore insérés. C'est donc tout naturellement, en pente douce malgré les traumatismes qu'ont pu créer les conquêtes danoise (à partir de 1013) et normande (à partir de 1066), qu'une Angleterre qui, vers l'an 1000, semble avoir été majoritairement organisée en communautés de paysans libres, a glissé vers le régime seigneurial ; et même alors, certains secteurs de l'eastern zone semblent être restés rétifs à ce mode d'organisation sociopolitique et ont conservé une forte proportion de ce qu'en France on appellerait des alleutiers et qu'en Angleterre on appelle des sokemen.

De telles conclusions, qui ne peuvent être atteintes qu'en accordant la primauté aux données archéologiques et en les confrontant aux autres sources, ne manqueront pas d'être discutées. Mais il convient dès maintenant de saluer l'ambition et l'intelligence du livre de John Blair : un grand livre qui fera date.

Alban Gautier (Université de Caen Normandie) 